International Journal of Fatigue, 30 (2008): 967-977

\title{
On the inverse power laws for accelerated random fatigue testing
}

\author{
G. Allegri* and X. Zhang \\ Aerospace Engineering Department \\ School of Engineering \\ Cranfield University \\ Cranfield, Bedfordshire, MK43 0AL, UK
}

\begin{abstract}
This paper addresses the usage of inverse power laws in accelerated fatigue testing under wide-band Gaussian random loading. The aim is not at predicting an absolute value of fatigue life but assessing the fatigue damage relative accumulation. The widely accepted inverse power scaling laws in fatigue damage assessment is discussed, reviewing the engineering standards and pointing out their inherent limitations. A physically consistent general scaling law is obtained by rigorous mathematical analysis in the framework of random vibration theory and the rules of safe-life fatigue analysis. Simplifications of the general scaling rule are presented, highlighting conditions under which the current standard practice could provide a correct an acceptable estimation of the relative fatigue damage accumulation.
\end{abstract}

Key words: fatigue, inverse power law, random loading, cycle counting, accelerated testing

\section{Nomenclature}

$T_{r}$ - fatigue life in the operative environment;

$T_{s}$ - fatigue life in the testing condition;

$g$ - acceleration root mean square value (RMS);

$g_{r}$ - acceleration RMS in the operative environment;

* Corresponding Author

Email address: g.allegri@cranfield.ac.uk (G. Allegri). 
$g_{s}$ - acceleration RMS in the testing environment;

$\bar{g}^{*}=\bar{g} / g$ - normalized mean value of the acceleration;

$\hat{g}^{*}=\hat{g} / g$ - normalized acceleration amplitude;

$\alpha$ - inverse power law exponent;

$\nu$ - frequency;

$S_{r}(\nu)$ - power spectral density (PSD) of the operative acceleration;

$S_{s}(\nu)$ - PSD of the testing acceleration;

$S_{x y}(\nu)$ - cross PSD associated with the time dependent variables $x$ and $y$;

$\lambda_{(r), p}-p$-th order spectral moment of the operative PSD;

$N_{(r), 0}$ - number of zero crossings with positive slope per unit time for the operative acceleration history;

$N_{(r), p}$ - number of peaks per unit time for the operative acceleration history;

$\tilde{N}_{0}$ - total number of zero crossings with positive slope;

$\sum_{i, j}^{R F}$ - rainflow matrix;

M - structural mass matrix;

C - structural damping matrix;

$\mathbf{K}$ - structural stiffness matrix;

$\mathbf{S}$ - matrix for expressing the von Mises stress in spectral form;

$F(t)$ - vector of external excitation forces;

$H_{s}(\nu)$ - harmonic response associated to the $s$-th mode

$\epsilon$ - vector representing the six independent component of the strain tensor;

$\sigma$ - vector representing the six independent component of the stress tensor;

$a$ - external acceleration vector; 
$\sigma_{e q}$ - von Mises equivalent stress;

$\tilde{\sigma}$ - RMS of the von Mises equivalent stress;

$b$ - Basquin's fatigue exponent;

\section{Introduction}

Several methodologies have been developed for predicting fatigue life of engineering components under random vibration loads. These methods may be divided into two main groups depending on the parameters and data used in the analysis. The time-domain based analysis, which depends mainly on the stress or strain amplitudes in the time history, has been well established (e.g. [1]-[3]) and widely accepted. In a time-domain approach, a cycle counting procedure and, e.g. the rainflow algorithm [4]-[5], a cumulative damage rule are employed. The only difference with the non-vibration applications is that random loading causes stress ranges of all magnitudes from zero to a set maximum, which must be specified via probability density distributions and the damage summation must be evaluated as an integral. However computer simulation of all possible stress-time history samples is very time consuming and at the design stages the full stress history may not be available.

On the other front, the frequency domain analyses are now an accepted practice [6]-[10]. These methodologies are based on expressing the fatigue life as a function of the spectral characteristics of the excitation. However, in spite of considerable efforts, no general analytical solution is yet available; damage accumulation in frequency domain is currently described by approximated laws. Most of the existing techniques are limited to the stationary Gaussian loading histories and there is no general agreement on how the effects of multi-axial loading on fatigue life can be accounted for.

Apart from random loading effects, many other random factors also influence fatigue damage accumulation, e.g. the randomness of material properties and randomness of defect distribution in components. It is recognized that the calculation of fatigue damage accumulation in absolute terms, i.e. life cycles, is still extremely difficult due to the randomness of the above factors. On the other hand, even for the simple constant or variable amplitude loading conditions, large scatters exist in fatigue life prediction. This is mainly due to the inherent limitation of the linear cumulative damage rule, i.e. the Miner's law, which is also widely employed in vibration fatigue analysis. Numerous statistical studies and fatigue tests have been conducted to find the statistical distribution of the damage [11]. 
This paper is not about proposing another method for predicting fatigue life. Rather, it aims at providing further insight into the inverse power scaling law models, which allow estimating the relative damage accumulation based on laboratory tests. It is customary to perform accelerated random vibration testing in a laboratory environment, which in terms of loading is considerably more severe than the operative one; the operative life duration is then estimated by relating the structural fatigue life tested in the laboratory condition by a proper scaling factor. The scaling law depends on the ratio of the load severities, e.g. using the root mean square values, of the two environments and also on some empirical or semi-empirical constants, which are determined from either the previous design or usage experience. A widely employed inverse power law in the aerospace industry to correlate the laboratory tests to the real service life is as follows [12]-[14]:

$$
\frac{T_{s}}{T_{r}}=\left(\frac{g_{r}}{g_{s}}\right)^{\alpha}
$$

where $T_{r}$ is the fatigue life in terms of time duration in an actual operative environment, $T_{s}$ the fatigue life corresponding to accelerated laboratory test, $g_{r}$ and $g_{s}$ are the root mean square (RMS) values of the actual and lab simulated accelerations (or loads), respectively, applied to the structures. The exponent $\alpha$ represents the slope of the material S-N curve in log-log coordinates. The inverse power scaling law in eq. (1) was derived from the Coffin-Manson's model of fatigue life [15]-[16]. It is worth stressing that eq. (1) is implemented in the MIL Standard 810 [17], which specifies the lab testing environment for the certification of military aerospace sub-systems in the US; this standard is also commonly adopted by countries belonging to the NATO. Eq. (1) simply states that the testing time in the laboratory environment is inversely proportional to the applied force RMS via exponent $\alpha$. Although it has been recognised that the exponent $\alpha$ is the slope of the material $\mathrm{S}-\mathrm{N}$ curve, the assumptions made to derive eq. (1) are extremely reductive for the following reasons

- Coffin-Manson's model [15] is strictly valid for a uni-axial applied force, i.e. a simple tensile/compressive stress, while eq. (1) is applied for the safe life estimation of complex structures, for which the operative loading and the resultant stress field may be multi-axial;

- complex structures may be made of several different materials, each having a characteristic sensitivity to fatigue damage accumulation;

- there is no rigorous proof that the acceleration RMS are sufficient to fully describe the random vibration environment, especially for wide-band excitation spectra; 
- under random loading the material S-N curve exponent is generally dependent on the spectral properties of the excitation [18]-[19]; standard fatigue tests on materials are performed with sinusoidal excitation, but the slope of the S-N curve for an assigned material changes under random loading. The methodology developed in this paper is based on considering the S-N curve under random loading condition

Therefore, in the light of the complexity of the phenomenon under investigation, the very simple scaling law in eq. (1) represents a very crude approximation of the fatigue behavior for complex structures, yet it is widely applied for safe life analysis, at least at the design stage. The characteristic exponent $\alpha$ usually ranges between 5 and 8, depending on the designer's background, previous experiences and validation tests. Basically each company operating in the aerospace industry works with values adjusted to its own design and testing database; this implies that very little of the original physical meaning of the $\alpha$ exponent is retained in engineering applications. Actually the scaling exponent appears to be an empirical factor which weights all the uncertainties and limitations related to the "tout-court" application of eq. (1), regardless to its pristine interpretation as the material S-N curve slope. Ref. [13] and [17] recommend that for eq. (1) to be valid the spectral response of the structure in the operative environment and the testing conditions must be "similar" in shape; unfortunately no quantitative means are provided to identify whether the recommended "similarity" holds or not and what sort of effects this might have on the $\alpha$ values to be employed in accelerated testing.

In this paper a physically consistent scaling law is obtained by means of a rigorous approach within the framework of random vibration theory [20] and the semi-empirical rules of the safe-life fatigue analysis; multi-axial wide-band loading is taken into account in the analysis. The objective is to provide a useful tool to estimate the relative accumulation of fatigue damage under complex random loading conditions and discuss in detail under which assumptions eq. (1) can be considered as a valid approximation for practical purposes.

\section{Problem statement and basic assumptions}

The derivation of a physically consistent scaling law for relative fatigue damage accumulation is based on the following assumptions

- the materials are linear elastic;

- no geometrical non-linearities arise (small strain elasticity);

- multi-axial loads are applied to the structure in the form of random sta- 
tionary Gaussian wide-band excitations;

- a safe life approach based on S-N curves is used;

- the von Mises equivalent stress can be used in standard material S-N curve in order to estimate the fatigue life duration due to multi-axial loading.

It is worth stressing that the von Mises equivalent stress mentioned in the last point above does represent a spectral based extension of the familiar scalar stress measure commonly exploited in static analysis; this extended definition is discussed in detail in Sec. 3.3.

\section{Analysis methodology}

The procedure to obtain the desired scaling laws starts from defining the spectral properties of the excitation and demonstrating that rainflow counting is not affected by the excitation level, provided that the input PSD shape stays the same. Describing the structure by a finite set of discretized Lagrange's equations, the spectral properties of the stresses attained are then expressed as functions of those featuring the excitations. Then a spectral representation of the von Mises equivalent stress is introduced; this is needed to obtain a scalar stress measure from the general stress tensor in the case of multi-axial loading. An exact inverse power scaling law is obtained by substituting the spectral representation of the equivalent von Mises stress in the S-N curve equation, i.e. the Basquin's equation [21].

\subsection{Spectral properties of the excitation}

Since the applied load is random, the acceleration RMS provides a measure of the overall energy level associated to the vibration environment. In the framework of the random vibration theory, if the excitation is stationary, the RMS represents the square root of the acceleration PSD, so that

$$
g_{r}=\sqrt{\int_{0}^{+\infty} S_{r}(\nu) d \nu}
$$

where $\nu$ is the frequency and $S_{r}(\nu)$ is the one-sided acceleration PSD of the actual excitation. An equivalent definition holds for the simulated RMS of acceleration $g_{s}$ expressed by $S_{s}(\nu)$. Therefore the problem of designing a testing environment which resembles the actual operative one can be solved by 
introducing a scaling factor between the PSD functions representing the actual acceleration $S_{r}(\nu)$ and the simulated one $S_{s}(\nu)$. This can be achieved by relating the PSD to some statistical parameters.

Assuming that the excitation is zero-mean valued, the $p$-th spectral moment is defined as follows

$$
\lambda_{(r), p}=\int_{0}^{+\infty} S_{r}(\nu) \nu^{p} d \nu
$$

The first order spectral moment $\lambda_{0}$ is simply the acceleration RMS squared. The average number of zero-crossings $N_{(r), 0}$ with positive slope per unit time featuring the actual load is given by

$$
N_{(r), 0}=\sqrt{\frac{\lambda_{(r), 2}}{\lambda_{(r), 0}}}
$$

and the average number $N_{(r), p}$ of peaks per unit time is defined as:

$$
N_{(r), p}=\sqrt{\frac{\lambda_{(r), 4}}{\lambda_{(r), 2}}}
$$

The bandwidth parameter is defined as the ratio of number of crossings to number of peaks per unit time

$$
\xi=\frac{N_{(r), 0}}{N_{(r), p}}
$$

If the actual and the experimental PSDs are related by the simple scaling relation

$$
S_{s}(\nu)=K_{s r} S_{r}(\nu)
$$

where $K_{s r}>1$ is a constant, all the spectral moments from eq. (3) are scaled by $K_{s r}$ and the average numbers of zero-crossings and peaks per unit time, as well as the bandwidth parameter, do not change.

\subsection{Rainflow counting for random loading}

The above result has important implications in terms of cycle counting by the rainflow method; it is well known that the rainflow algorithm, even in its 
non-recursive formulation, does not allow to obtain closed form solutions for the cumulative distributions of cycle amplitude and mean values.

Let $\bar{g}_{i}^{*}$ and $\hat{g}_{j}^{*}$ be of the non-dimensional mean acceleration $\bar{g}^{*}=\bar{g} / g$ and nondimensional acceleration amplitude $\hat{g}^{*}=\hat{g} / g$ as obtained by rainflow counting from a random acceleration history having PSD $S(\nu)$ and RMS $g$. Each of the partitions comprises $M$ levels. For a detailed discussion of accurate and efficient rainflow counting methodologies the reader is referred to [5].

Let $\Sigma^{R F}$ be the rainflow matrix obtained by the acceleration cycle counting; each entry $(i, j)$ of the matrix is given by

$$
\Sigma_{i, j}^{R F}=\frac{n_{i, j}}{N}
$$

where $n_{i, j}$ represents the number of cycles whose mean value and amplitude satisfy

$$
\bar{g}^{*} \in\left[\bar{g}_{i-1}^{*}, \bar{g}_{i}^{*}\right) \cap \hat{g}^{*} \in\left[\hat{g}_{j-1}^{*}, \hat{g}_{j}^{*}\right)
$$

and $N$ is the total number of cycles counted. It is clear that for $N \rightarrow \infty$ each entry $(i, j)$ of the rainflow matrix represents the probability of finding in the load history acceleration cycles with mean value and amplitude satisfying (9). In standard practise the rainflow ranges are not normalized by the acceleration RMS, but employing the non-dimensional mean value and amplitude guarantees that the rainflow matrix is invariant with respect to the scaling (7) of the original PSD function. This can be proved by observing that a time history having a PSD $S(\nu)$ can be converted to a time history having the PSD $K S(\nu)$, where $\mathrm{K}$ is a positive constant, by multiplying all the $g$ values by $\sqrt{K}$. If $g$ is the root mean square of the pristine time history, $g \sqrt{K}$ would be the root mean square of the modified one. This does not affect the number of zerocrossings and peaks as well as the sequence of turning points that represents the input for the rainflow counting; the cycles mean values and amplitude will be all multiplied by $\sqrt{K}$ for the modified time history, so this would not affect the rainflow matrix $\Sigma^{R F}$.

Therefore the conclusion is that for stationary Gaussian stochastic processes which have PSD functions with the same shape, i.e. the PSD of the different process can be obtained by a proper scale factor $K>0$, the rainflow matrix is the same, provided that the mean value and amplitude levels are normalized by the root mean square value of the time history, i.e. the zero order spectral moment of the various PSD functions. The result just obtained is valid for a uni-axial random loading; in multi-axial loading condition it has been recognised that the damage accumulation in metals is essentially shear driven [22]. Small cracks can nucleate and growth on a "critical" plane where the maximum shear strain is attained; therefore the multi-axial damage assessment can be reduced to an uni-axial equivalent problem. Nevertheless normal stresses and shear strains can occur out of phase [23] and this makes cycle 
counting more difficult; moreover the orientation of the critical plane is generally unknown so, even if a proper counting algorithm is available, the damage assessment procedure must be repeated for several candidate critical orientations. Improved multi-axial cycle counting algorithms have been proposed [23]-[24] in order to cope with the effects mentioned above. In this paper the critical plane cycle counting is not considered because a scalar stress measure is introduced via a spectral representation of the von Mises stress; this allows reducing the multi-axial cycle counting to the much simpler random uni-axial scenario discussed above.

\subsection{Derivation of equivalent stress spectrum}

The main problem in applying eq. (1) to assembled structures is relating the local stress/strain state in each component to the external acceleration spectrum. This task requires a complete model of the structure. Assuming that this model is somehow available, for example in a discrete form, the PSD of the local stress/strain field can then be obtained from the acceleration and the natural vibration modes of the assembled structure; it is here assumed that the structure is discretized by applying the FE method. Let the dynamics of the structure be represented by the following $n$-degrees of freedom second order system

$$
\mathbf{M} \ddot{x}(t)+\mathbf{C} \dot{x}(t)+\mathbf{K} x(t)=F(t)
$$

where $x(t)$ is the vector of nodal displacements, i.e. the degrees of freedom, $\mathbf{M}$ is the mass matrix, $\mathbf{C}$ the damping matrix, $\mathbf{K}$ the stiffness matrix and $F(t)$ the vector of external force applied. It is here assumed that the dynamic analysis of the system is performed by the well-established large mass technique [25]; this method is based on placing a "seismic" mass, whose magnitude is much larger than that featuring the structure, at the discretization nodes for which known accelerations are prescribed. Large mass elements act effectively as constraints for the structural nodes, enforcing the motion prescribed by the user; this method is the simulation counterpart of experimental vibration tests performed by shakers. The large mass approach is suitable for both harmonic, transient and random response analyses. The FE analysis of structural random vibration in the framework of linear elasticity and small strain assumption allows expressing the stress response PSD matrix $\mathbf{S}_{\sigma \sigma}^{(e)}(\nu)$ as a function of the input acceleration PSD matrix $\mathbf{S}_{a a}(\nu)$ via the following equation

$$
\mathbf{S}_{\sigma \sigma}^{(e)}(\nu)=\mathbf{D}^{(e)}(x, y, z) \mathbf{U}(\nu) \mathbf{G S}_{a a}(\nu) \mathbf{G}^{T}
$$

The superscript $(e)$ in eq. (11) denotes any arbitrary element belonging the the body/structure discretization; the matrix $\mathbf{D}^{(e)}(x, y, z)$ contains the elemental 
material properties and shape functions employed in the discretization. The matrix $\mathbf{U}(\nu)$ comprises the harmonic response of the structural degrees of freedom. Finally the matrix $\mathbf{G}$ converts the seismic mass accelerations to the nodal loads on the right hand side of eq. (10). The details of eq. (11) derivation are presented in Appendix A for sake of completeness.

The problem now is how to relate eq. (11) to the fatigue damage accumulation. The strain-stress field is multi-axial, so the conventional uni-axial fatigue data cannot be applied directly, unless the structural configuration is extremely simple. Secondly for a multi-axial stress/strain history it is also necessary to apply a modified rainflow counting algorithm, basically taking into account all the possible linear combination of the stress/strain history components for a discrete set of geometrical points belonging to the structure. These latter can be either the FE mesh nodes or a suitable set of collocation points chosen within the elements or both these things. It is clearly understood that generating random stress histories with proper auto and cross-correlation properties, combining them, rainflow counting for a discrete set of collocation points and finally evaluating the damage by a multi-axial fatigue accumulation criterion would require a tremendous amount of computational time; this happens even for relatively simple configurations. Moreover several multi-axial fatigue criteria have been proposed and choosing the right one for the specific application is not easy. However it must be pointed out that the main aim here is to establish proper scaling factors between laboratory tests and the actual operative environment. So the requirement for the multi-axial fatigue criterion is not to be accurate in absolute terms, but only to provide a consistent representation in the physics of fatigue damage accumulation in order to compare the effects of different excitation histories.

Several multi-axial fatigue accumulation models for metals are based on the von Mises stress concept [26]-[27]; this approach is appealing since a multi-axial stress state can be converted to an equivalent uni-axial one and the standard safe life calculations can be performed by entering the S-N curves with the von Mises equivalent stress. Multi-axial versions of the Goodman equivalent stress amplitude have been expressed in terms of the hydrostatic stress components. Low cycle fatigue models have been developed by considering the von Mises equivalent strain and hydrostatic stresses in order to model the mean value effect.

The von Mises equivalent stress is defined as follows

$$
\sigma_{e q}^{2}=\sigma_{x}^{2}+\sigma_{y}^{2}+\sigma_{z}^{2}-\sigma_{x} \sigma_{y}-\sigma_{x} \sigma_{z}-\sigma_{y} \sigma_{z}+3 \tau_{x y}^{2}+3 \tau_{x z}^{2}+3 \tau_{y z}^{2}
$$

From Ref. [27], introducing the $\mathbf{S}$ matrix, the von Mises stress can be expressed in the following equivalent form

$$
\sigma_{e q}^{2}=\operatorname{Tr}\left[\mathbf{S} \sigma \sigma^{T}\right]
$$


where $\operatorname{Tr}$ is the trace operator and $\mathbf{S}$ is given by

$$
\mathbf{S}=\left(\begin{array}{cccccc}
1 & -\frac{1}{2} & -\frac{1}{2} & 0 & 0 & 0 \\
-\frac{1}{2} & 1 & -\frac{1}{2} & 0 & 0 & 0 \\
-\frac{1}{2} & -\frac{1}{2} & 1 & 0 & 0 & 0 \\
0 & 0 & 0 & 3 & 0 & 0 \\
0 & 0 & 0 & 0 & 3 & 0 \\
0 & 0 & 0 & 0 & 0 & 3
\end{array}\right)
$$

The main problem in applying the von Mises equivalent stress to the random vibration fatigue damage is that, for a stationary Gaussian stress history, $\sigma_{e q}$ is not normally distributed; this implies that the von Mises stress distribution should be obtained by a direct time-domain Monte Carlo simulation, which is very demanding from a computational point of view. The definition (13) allows overcoming this problem; taking the expectation of both sides of (13) yields

$$
E\left[\sigma_{e q}^{2}\right]=\operatorname{Tr}\left[\mathbf{S C}_{\sigma \sigma}\right]
$$

where $\mathbf{C}_{\sigma \sigma}=E\left[\sigma \sigma^{T}\right]$ is the covariance matrix of the stress vector. Now, since the PSD is the Fourier transform of the correlation function, for each element $e$ of the structural FE mesh the stress covariance matrix can be obtained as

$$
\mathbf{C}_{\sigma \sigma}^{(e)}=\int_{0}^{+\infty} \mathbf{S}_{\sigma \sigma}^{(e)}(\nu) d \nu
$$

where the argument of the integral is given by (11). Thus from (15) one obtains

$$
\left(E\left[\sigma_{e q}^{2}\right]\right)^{(e)}=\operatorname{Tr}\left[\mathbf{S} \int_{0}^{+\infty} \mathbf{S}_{\sigma \sigma}^{(e)}(\nu) d \nu\right]
$$

Following Ref. [27], one can postulate that there exists a von Mises equivalent stress whose mean quadratic value in expressed as the integral of the associated power spectral density function over the whole frequency range as follows

$$
\left(E\left[\sigma_{e q}^{2}\right]\right)^{(e)}=\int_{0}^{+\infty} S_{\sigma_{e q} \sigma_{e q}}^{(e)}(\nu) d \nu
$$


Comparing eqs. 17 and 18 one can define the equivalent Von Mises stress as the stochastic process whose PSD function is given by

$$
S_{\sigma_{e q} \sigma_{e q}}^{(e)}(\nu)=\operatorname{Tr}\left[\mathbf{S S}_{\sigma \sigma}^{(e)}(\nu)\right]
$$

The definition in eq. (19) shows that the equivalent von Mises stress is a stationary Gaussian process whose spectral representation is obtained directly from that worked out for the cross-PSD of the stress components [27]. This result establishes a direct link between the accelerations applied to the structure and the von Mises stress levels attained in each element $e$. If the excitation has zero mean value, then the von Mises stress also has the same property; in the case of a one-dimensional problem the von Mises stress yields simply the only stress component featuring the structural response.

By introducing the spectral definition of the von Mises stress (18) there is no more necessity of simulating the stress histories for each stress tensor component; these simulations can now be performed directly by generating a von Mises stress history having the PSD (19), which is immediately related to the output (11) of a random vibration FE analysis. Moreover there is no need for multi-axial rainflow counting, since the three-dimensional problem is now represented by a one-dimensional variable, that is the von Mises stress; the counting procedure can be applied directly to $\sigma_{e q}$ for each element.

\subsection{General Scaling law}

The random vibration response of a continuous complex structure has been reduced to that featuring the discrete system which can be obtained by applying the FE method. The von Mises stress has been chosen as the representative variable ruling the damage accumulation in a three-dimensional stress state, thus leading to a one-dimensional of fatigue life assessment. the fatigue life of all the materials employed in the structure can be predicted by a $\mathrm{S}-\mathrm{N}$ curve based safe-life approach. The choice of the statistical parameters by which the random fatigue experimental data can be expressed is not trivial; the basic S$\mathrm{N}$ curves for deterministic constant amplitude loading are presented as stress amplitude versus the number of cycles. For a random load history the number of cycles cannot be identified "a priori", but it must be counted by the rainflow algorithm. Both the number of zero-crossings with positive slope and the number of peaks provide indirect measures of the total number of cycles to which the tested specimens undergo, but the bandwidth parameter also has a considerable influence on the accumulated damage.

From a qualitative point of view this can be understood by observing that, for a narrow-band stress history, the number of peaks is close to that of zerocrossings, so the cycle measure tends to be unique; on the other hand for a 
wide band process the numbers of zero crossings and peaks differ considerably, being the former much less than the latter. A wide-band load history is expected to produce more "rainflow" fatigue cycles than zero-crossings with positive slope, because the stress values tends to be clustered in sequences having a non-zero mean value; therefore for each zero-crossing there is a certain number of peaks and troughs, some of which identify hysteretic loops for the material, i.e. fatigue cycles according to the rainflow algorithm.

Keeping these differences in mind, it is here stated to measure the fatigue life duration by the total number of crossings with positive slope; therefore the S$\mathrm{N}$ curves can be expressed in terms of the root mean square value $\tilde{\sigma}$ of the von Mises $\sigma_{e q}$ stress featuring a random load history, versus the total number of zero crossing with positive slope $\tilde{N}_{0}$. Thus the fatigue strength in multi-axial fatigue accumulation can be expressed by a Basquin's type equation [21]

$$
\tilde{\sigma}=C \tilde{N}_{0}^{-1 / b}
$$

The assumption that the stress measure is represented by the RMS of the load history is useful because an RMS value can be also introduced for deterministic load histories. For example the RMS of a sinusoidal signal is its amplitude divided by $\sqrt{2}$; for a sawtooth signal the scaling factor is $\sqrt{3}$; finally for a square wave signal the amplitude and RMS are exactly the same.

Note that this S-N curve in eq. 20 is not necessary that obtained by sinusoidal loading with constant amplitude; the effects of load interactions which occurs in a random stress history increase the $\mathrm{S}-\mathrm{N}$ curve slope with respect to the sinusoidal load case [30]. Anyway the specific PSD shape parameters do not appear in (20). This means that (20) is assumed to be valid for both narrowband and wide-band processes; this point will be discussed more in detail later on.

The root mean square value of the von Mises stress for each element is obtained by taking the square root of both eq. (17) members, yielding

$$
\tilde{\sigma}^{(e)}=\sqrt{\operatorname{Tr}\left[\mathbf{S} \int_{0}^{+\infty} \mathbf{S}_{\sigma \sigma}^{(e)}(\nu) d \nu\right]}
$$

Again (21) is directly linked to the stress cross-PSD (A.17). Assuming to consider the actual operative vibration environment $(r)$ and the simulated laboratory one $(s)$ the ratio of the fatigue durations in terms of crossings with positive slope is obtained by (20) as

$$
\frac{\tilde{N}_{0,(s)}^{(e)}}{\tilde{N}_{0,(r)}^{(e)}}=\left(\frac{\tilde{\sigma}_{(r)}^{(e)}}{\tilde{\sigma}_{(s)}^{(e)}}\right)^{b^{(e)}}
$$


where $b^{(e)}$ is the exponent of Basquin's equation [i.e. eq. (20)] for the material the element is made of.

If $\tilde{T}_{(r)}=\tilde{N}_{0,(r)} / N_{0,(r)}$ is the actual time to failure and $\tilde{T}_{(s)}=\tilde{N}_{0,(s)} / N_{0,(s)}$ is the laboratory time to failure, eq. (22) leads to

$$
\frac{\tilde{T}_{(s)}^{(e)}}{\tilde{T}_{(r)}^{(e)}}=\frac{N_{0,(r)}^{(e)}}{N_{0,(s)}^{(e)}}\left(\frac{\tilde{\sigma}_{(r)}^{(e)}}{\tilde{\sigma}_{(s)}^{(e)}}\right)^{b^{(e)}}
$$

Exploiting (4) one can write

$$
\frac{N_{0,(r)}^{(e)}}{N_{0,(s)}^{(e)}}=\sqrt{\frac{\lambda_{(r), 2}^{(e)} \lambda_{(s), 0}^{(e)}}{\lambda_{(s), 2}^{(e)} \lambda_{(r), 0}^{(e)}}}
$$

where the spectral moments $\lambda_{(r), p}$ and $\lambda_{(s), p}$ are given by (3) and they are referred to the equivalent von Mises stress for the $e$-th element. Since the square root of the zero-th order spectral moment is simply the root mean square value of the stochastic process, substituting (24) into (23) yields

$$
\frac{\tilde{T}_{(s)}^{(e)}}{\tilde{T}_{(r)}^{(e)}}=\sqrt{\frac{\lambda_{(r), 2}^{(e)}}{\lambda_{(s), 2}^{(e)}}}\left(\frac{\tilde{\sigma}_{(r)}^{(e)}}{\tilde{\sigma}_{(s)}^{(e)}}\right)^{b^{(e)}-1}
$$

Finally substituting eqs. (21) and (3) in the last equation yields

$$
\frac{\tilde{T}_{(s)}^{(e)}}{\tilde{T}_{(r)}^{(e)}}=\sqrt{\frac{\operatorname{Tr}\left[\mathbf{S} \int_{0}^{+\infty} \mathbf{S}_{\sigma \sigma, r}^{(e)}(\nu) \nu^{2} d \nu\right]}{\operatorname{Tr}\left[\mathbf{S} \int_{0}^{+\infty} \mathbf{S}_{\sigma \sigma, s}^{(e)}(\nu) \nu^{2} d \nu\right]}}\left\{\frac{\operatorname{Tr}\left[\mathbf{S} \int_{0}^{+\infty} \mathbf{S}_{\sigma \sigma, r}^{(e)}(\nu) d \nu\right]}{\operatorname{Tr}\left[\mathbf{S} \int_{0}^{+\infty} \mathbf{S}_{\sigma \sigma, s}^{(e)}(\nu) d \nu\right]}\right\}^{\frac{b^{(e)}-1}{2}}
$$

Eq. (26) gives the exact time scale for an arbitrary linear elastic structure subjected to a stationary Gaussian excitation; the cross-PSD of the local stress field for both the operative and simulated environments is given by (11). Since the equivalent von Mises stress has been employed to predict the fatigue damage according to the Basquin's type equation (20), the applicability of (26) is restricted only to metallic structures. Other class of materials will require introducing different damage indexes; the procedure here outlined is general, provided that those alternative damage indexes admit a spectral representation similar to that employed for the von Mises stress. For example it can be easily demonstrated that standard failure criteria for composite materials (i.e. Tsai-Hill, Tsai-Wu, Hoffman, etc.) admit a spectral representation analogous to that in eq. (18).

The scaling law (26) is valid for all the elements comprised in the FE model 
of the complete structure; the proper scaling time can be evaluated by the elements which under the prescribed vibration environment are subjected to the largest von Mises stress RMS and which are the most susceptible to accumulate fatigue damage.

\subsection{Simplification of the general scaling law}

A fundamental question arises at this point: how does (26) relate to the empirical scaling law in eq. (1)? In order to answer to this question the following assumptions are introduced:

1. the structure for laboratory testing is identical to that in operative conditions;

2. the constraints applied in the testing configuration and in the operative environment are exactly the same;

3. the cross-PSD of the accelerations applied to the testing structure are simply scaled with respect to the actual operative ones, i.e.

$$
\mathbf{S}_{a a,(s)}(\nu)=K_{s r} \mathbf{S}_{a a,(r)}(\nu)
$$

where $K_{s r}>1$ in order to accelerate the laboratory trials.

Under these assumptions, exploiting (27) and (11) one can write

$$
\mathbf{S}_{\sigma \sigma,(s)}^{(e)}(\nu)=K_{s r} \mathbf{S}_{\sigma \sigma,(r)}^{(e)}(\nu)
$$

Therefore the stress cross-PSD for the $e$-th element in the simulated lab vibration environment are simply scaled by the factor $K_{r s}$ with respect to the operative ones. Thus substituting (28) into (26) yields

$$
\frac{\tilde{T}_{(s)}^{(e)}}{\tilde{T}_{(r)}^{(e)}}=\left(\frac{1}{K_{s r}}\right)^{b^{(e)} / 2}
$$

The scale factor $K_{s r}$ must be a scalar, i.e it must be the same for all the cross-PSD, otherwise eq. (29) will not be applicable. If the excitation is onedimensional, (27) reduces to

$$
S_{a a,(s)}(\nu)=K_{s r} S_{a a,(r)}(\nu)
$$


where $S_{a,(r)}$ and $S_{a,(s)}$ are now scalar functions representing the auto-PSD of the only external excitation applied. Taking the integral over the real positive axis of both members of the last equation yields

$$
g_{(s)}^{2}=K_{s r} g_{(r)}^{2}
$$

where $g_{(s)}$ and $g_{(r)}$ are the RMS of the simulated and actual one-dimensional accelerations. Substituting (31) into (29) leads to

$$
\frac{\tilde{T}_{(s)}^{(e)}}{\tilde{T}_{(r)}^{(e)}}=\left(\frac{g_{(r)}}{g_{(s)}}\right)^{b^{(e)}}
$$

Therefore eq. (32) demonstrates that the commonly adopted inverse power scaling law (1) is just a particular case of the exact expression in eq. (26). The simplification here demonstrated is valid only under the assumptions listed above in the case of multi-axial loading. The critical point is that (1) is strictly valid for arbitrary structural configuration only if the excitation PSD shape is the same passing from the testing environment to the operative one; moreover, strictly speaking, the structural geometrical configurations must be identical, as well as the constraints applied. Any violation of the assumptions made will force to employ eq. (26) in order to get the correct time scaling.

Nevertheless, if the general scaling law from eq. (26) is applied, particular care must be taken to avoiding substantial changes of the geometry/constraint layout; for example by removing constraints it is possible to make spurious vibration modes appear, so that the estimation of the scaling factors will be too conservative. On the other hand, if additional constrains are added in the testing environment, some vibration modes featuring the operative scenario will be suppressed and the actual scaling factor underestimated.

\section{S-N curves in a random vibration environment}

In the above sections it has been demonstrated how an exact scaling law, i.e. eq. (26), for accelerated fatigue testing can be obtained in the framework of random vibration theory, assuming that the relative damage accumulation due to multi-axial load can be quantified by the equivalent von Mises stress. In the special case where the testing input spectra and the operative ones have the same shape, the exact expression can be simplified, leading to eq. (32), which is identical to the widely employed inverse power law from eq. (1). It is also recognised that the characteristic exponent $\alpha$ is the material $\mathrm{S}-\mathrm{N}$ curve slope in a log-log representation of the fatigue life versus the applied stress RMS. The key point at this stage is to understand how to estimate $b^{(e)}$ for either the 
exact or the simplified scaling laws; the basic issue is that the S-N curve exponent as obtained from standard constant amplitude fatigue tests on materials is not directly applicable to eqs. (26) and (32). This is due to the fact that random load histories are deeply affected by load interaction and sequence effect; the impact of those effects in reducing the actual fatigue life for variable amplitude loading is well documented in literature [28]. It has been demonstrated [29]-[30] that load interaction effect for variable amplitude stress histories can be accounted for by a clockwise rotation of the S-N curves in the stress-cycle plane. This implies that the $\mathrm{S}-\mathrm{N}$ curve slope for variable amplitude loading must be increased with respect to constant amplitude conditions, yielding a shorter fatigue life for the same average stress amplitude.

To prove this point one can refer to a detailed survey of random fatigue accumulation in the aluminium alloy 2024-T4 [18]. This experimental survey was intended to find the effect of random load on the damage accumulation for the aforementioned alloy.

Six different PSD shapes were considered, having the general form

$$
\frac{S_{\sigma \sigma}}{K_{i}}=A_{i} \nu^{\beta}
$$

where $\sigma$ is the uni-axial stress applied, $\nu$ the frequency, $A_{i}$ and $\beta$ are shape parameters; $K_{i}$ is a further set of constants which were used to tailor the overall RMS value of the applied stress. A summary of the PSD function properties used during the laboratory tests is presented in tab. 1, where $N_{0}$ is the average number of zero-crossing with positive slope per unit time, $N_{p}$ the average number of peaks per unit time and $\xi$ is the bandwidth parameter. In all the tests the underlying stochastic process was Gaussian distributed with zero mean value. The RMS of the load history have been varied together with the basic PSD shapes. These latter fell in three different types, grouped according to their specific frequency dependence, as summarized in tab. 1; the PSD belonging to each type can be further distinguished by their bandwidth. A summary of the S-N data presented in [18] is shown in fig. (1). In this figure the RMS of the applied stress to failure is plotted versus the total number of zero crossing with positive slope; all the data from the random load histories have been grouped together and fitted by a single trend-line. The first observation is that, despite the different bandwidths of the stress histories, all the random fatigue data are well approximated by a single S-N curve. This latter appears to be shifted and rotated clockwise with respect to that featuring the sinusoidal load condition, thus resulting in a faster accumulation of fatigue damage under the random stress histories; however the net effect of different PSD shapes appears to be negligible. Considering that the bandwidth parameter for the six different PSD here considered ranges between 0.5 and 0.9 , the data in fig. (1) suggest that the damage accumulation in random fatigue depends only on the stress RMS and number of zero-crossings with positive slope. This conclusion is quite intriguing, since it suggests that 
for a random loading environment there is a unique S-N curve featuring the fatigue damage accumulation, which depends essentially on the material; the statistical information needed to plot the characteristic random S-N curve are the stress RMS and the total number of zero-crossings with positive slope.

Care must be taken in applying the previous conclusion to metallic alloys in general; the data available have been obtained for a specific aluminium alloy, namely Al 2024-T4. In Ref. [31] the fatigue accumulation in steel joints under wide-band excitation was considered; both Gaussian and non-Gaussian random loads were employed, with unimodal and bimodal spectra. It was demonstrated that non-Gaussian load histories accelerate the fatigue damage accumulation, so the S-N curve slope is dependent on the probability density function featuring the stochastic process which represents the applied load. Nevertheless, considering the case of Gaussian loading, the authors point out that increasing the bandwidth parameter has a limited effect on the fatigue life estimated in terms of number of zero-crossings with positive slope; this is due to the fact that, in wide-band processes, the peaks tend to correspond to small amplitude cycles which have a limited impact on the accumulated damage. Therefore the results in Ref. [31] support the conclusion that the S-N curves obtained for various bandwidth parameters in the case of a Gaussian excitation are close enough to be superposed, as already pointed out by the data from [18]; a definitive proof of this conclusion should be obtained by plotting the S-N curves as a function of the number of zero crossings with positive slope, whereas in Ref. [31] the number of rainflow cycles is considered. Similar remarks are also valid for the experimental tests performed in Ref. [32] on steel structures under random loading.

All the experimental results discussed above clearly point out that the fatigue exponent $b^{(e)}$ changes sharply passing from constant amplitude loading to random stress histories; therefore a correct scaling factor between a laboratory environment and the actual operative conditions can be established only if random fatigue data in a standard $\mathrm{S}-\mathrm{N}$ format are available for the structural material. The fact that $b^{(e)}$ appears to be not affected by the spectral properties of the random excitation could reduce the number of experimental tests to be carried out, but the physical consistency of this result for different structural materials must still be demonstrated.

\section{Conclusions}

The certification of aerospace structures in random vibration environments is based on accelerated fatigue testing. Scaling laws are needed to relate the test time in a laboratory environment to the actual operative life duration. Current certification methodologies are based on inverse power laws obtained from classical fatigue models, which are strictly valid only for constant ampli- 
tude uni-axial loading.

An exact scaling law has been obtained in the framework of classical random vibration theory and safe-life design methodologies; this scaling rule allows comparing the relative damage accumulation in vibration environments whose characteristic input spectra can be different, but the underlying probability distribution must be Gaussian. The exact scaling law has been obtained by assuming that the relative damage caused by multi-axial loading can be quantified by a scalar stress measure, namely the equivalent von Mises stress.

In the special case where the input PSD shapes featuring the testing environment and the operative one have the same shape, i.e. the input spectra are simply scaled by a constant factor, the general scaling rule is simplified to the semi-empirical inverse power law which is prescribed by the current certification standards.

The scaling law exponent which appears in both the exact and simplified scaling rules is that featuring the material $\mathrm{S}-\mathrm{N}$ curve; this had already been demonstrated for uni-axial loading cases, but the result has here been extended to the case of random multi-axial loading.

Nevertheless it must be considered that the material S-N curve obtained from a random stress history differs from that worked out employing a constant amplitude load. Generally speaking the fatigue accumulation in a random vibration environment is quicker than for constant amplitude loading, i.e. the $\mathrm{S}-\mathrm{N}$ curve slope is larger in the case of random loading. This implies that the correct value of the exponent which appears in the inverse power scaling law should be estimated by random fatigue tests on representative material samples. If the S-N curve characterising the fatigue damage accumulation under random loading is expressed as stress amplitude RMS versus number of zero crossings, some literature data actually suggest that the change in the $\mathrm{S}-\mathrm{N}$ curve slope is only a weak function of the bandwidth featuring the stress history. The aforementioned data were obtained for an aluminium alloy subjected to Gaussian load histories with different PSD shapes. This would imply that a correct scaling exponent can be obtained by only a limited amount of random fatigue tests, without the need of investigating the effects of several bandwidth values. More experimental data are needed to support this still very limited experimental evidence.

\section{Acknowledgements}

The authors are grateful to the UK Ministry of Defence for supporting this project under the research programme "Whole Life Assessment". We also acknowledge D. Charles and his colleagues at Cranfield Aerospace Ltd. for the helpful discussions. 
Type Function

$$
\nu_{\min }(H z) \quad \nu_{\max }(H z) \quad \mathbf{N}_{\mathbf{0}}\left(s^{-1}\right) \quad \mathbf{N}_{\mathbf{p}}\left(s^{-1}\right) \quad \xi
$$

\begin{tabular}{lllllll}
1 & $S_{\sigma \sigma} / K_{3}=10^{-3} \nu^{2}$ & 10 & 57 & 44 & 48 & 0.918 \\
2 & $S_{\sigma \sigma} / K_{2}=1$ & 10 & 70 & 44 & 54 & 0.801 \\
3.1 & $S_{\sigma \sigma} / K_{1}=1.6 \times 10^{3} \nu^{-2}$ & 20 & 100 & 44 & 64 & 0.691 \\
3.2 & $S_{\sigma \sigma} / K_{1}=5.8 \times 10^{3} \nu^{-2}$ & 43 & 100 & 65 & 73 & 0.892 \\
3.3 & $S_{\sigma \sigma} / K_{1}=10^{3} \nu^{-2}$ & 10 & 55 & 23 & 35 & 0.653 \\
3.4 & $S_{\sigma \sigma} / K_{1}=4 \times 10^{3} \nu^{-2}$ & 20 & 200 & 63 & 122 & 0.517 \\
\hline \hline
\end{tabular}

Table 1

PSD functions for random fatigue tests in Ref. [18]; the values $K_{i}$ are varied in order to attain different stress RMS levels for the same base PSD functions (i.e. PSD "shapes"). 


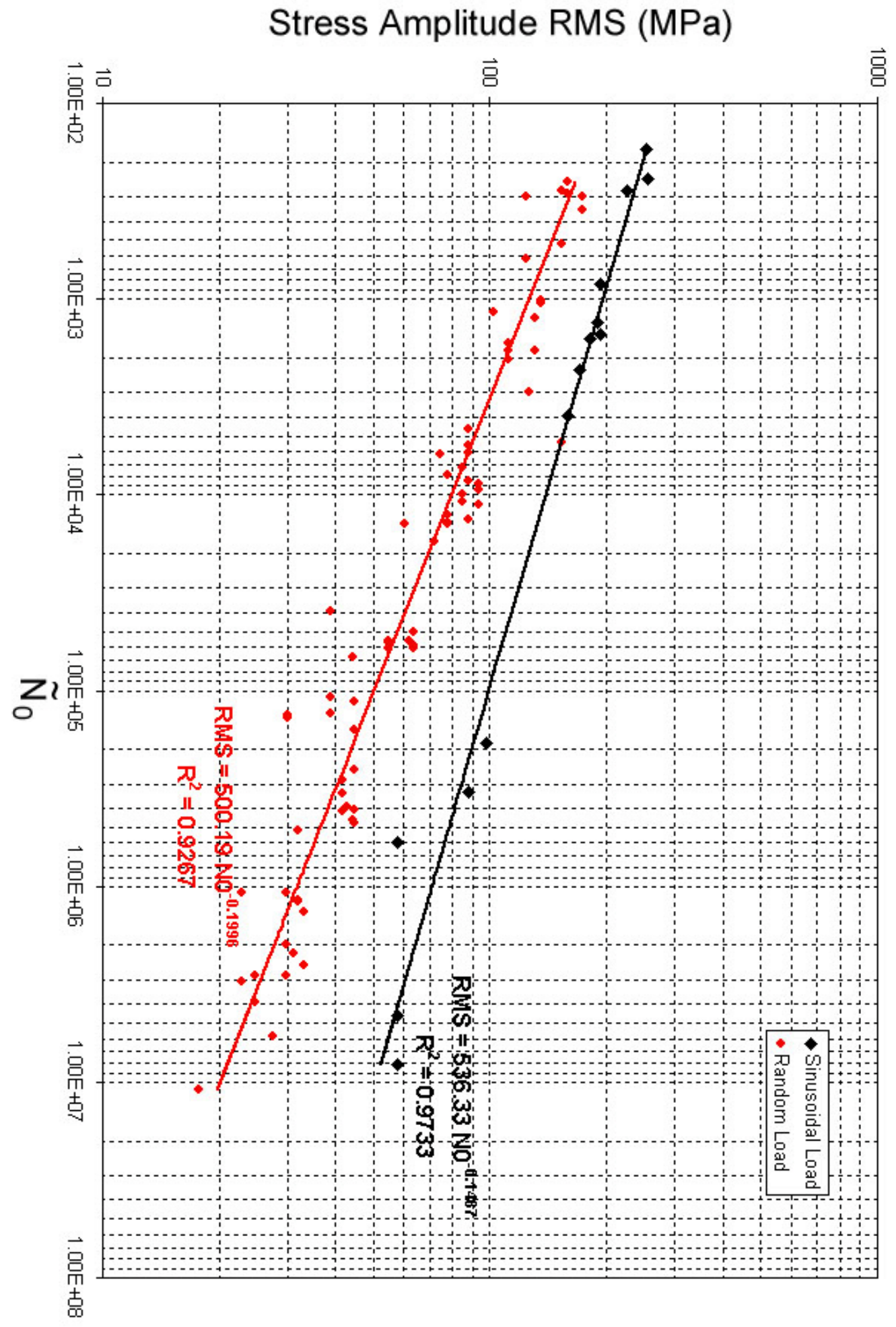

Fig. 1. S-N curves for Al 2024-T4 from Ref. [18]. 


\section{References}

[1] Sobczyk, K., Spencer, B. F., Random Fatigue: from data to theory. Academic Press, 1991.

[2] Dowling, N.E., Fatigue failure prediction of complicated load stress-strain histories ASTM J. Mater., 7, pp. 71-87, 1972.

[3] Wirshing, P.H., Haugen, E.B., Probabilistic design for random fatigue loads Journal of Engineering Mechanics Division, ASCE, 99, pp. 1593-1607, 1973.

[4] Rychlik, I., A new definition of the rainflow counting method Int J. Fatigue, 9, pp. 119-21, 1987.

[5] ASTM E-1049 -Standard Practices for Cycle Counting in Fatigue Analysis.

[6] Tovo, R., Cycle distribution and fatigue damage under wide-band random loading Int. J. Fatigue, 24, pp. 1137-47, 2002.

[7] Petrucci, G., Zuccarello, B., Fatigue life prediction under wide band random loading. Fatigue Fract. Engng. Mater. Struct., 27, pp. 1183-95, 2004.

[8] Benasciutti, D., Tovo, R., Spectral methods for lifetime prediction under wideband stationary random processes. Int. J. Fatigue, 27, pp. 867-877, 2005.

[9] Dirlik T., Application of computers to fatigue analysis. PhD Thesis, Warwick University, 1985.

[10] Sherratt, F., Bishop, N.W.M., Dirlik T., Predicting fatigue life from frequency domain data. Engineering Integrity, Vol. 18, pp. 12-16, Sept. 2005.

[11] Schijve, J., Statistical distribution functions and fatigue of structures. Int. J. Fatigue., 27, pp. 1031-39, 2005.

[12] Caruso, H., An Overview of Environmental Reliability Testing. Proceedings IEEE Annual Reliability and Maintainability Symposium, 0-7803-3112-5, 1996

[13] Caruso, H., Dasgupta, A., A fundamental overview of accelerated testing analytical models. Journal of the IEST, January/February, pp. 16-20, 1998.

[14] Farrar, C.R., Duffey, T.A., Cornwell, P.J., and Bement, M.T., Review of Methods for Developing Accelerated Testing Criteria. Proc. of the 17th International Modal Analysis Conference, Kissimmee, FL, pp. 608-614, Feb. 1999 .

[15] Manson, S., Fatigue: a complex subject - some simple approximations. Experimental Mechanics, 5, pp. 193-226, 1965.

[16] Coffin, L. F. Jr., A study of the effects of cyclic thermal stresses on a ductile metal. Trans. of ASME, 76, pp. 931-50, 1954.

[17] US Department of the Air Force, Military standard environmental test methods for aerospace and ground equipment, MIL-STD-810 C-F, Method 514: Vibration, June $14^{\text {th }}, 1962$. 
[18] Clevenson, S. A., Steiner, R., Fatigue life under random loading for several power spectral shapes. NASA-TR-266, Langley Station, Hampton, VA, 1967.

[19] Kouta, R., Guingand, M., Play, D., Design of welded structures working under random loading Proceedings of the IMechE Part K, Journal of Multi-body Dynamics, 216(2), pp. 191-201, 2002

[20] Meirovitch, L., Elements of Vibration Analysis, 2nd ed. McGraw-Hill, New York, 1986.

[21] Basquin, O.H., The exponential law of endurance tests. American Society for Testing and Materials, 10, pp. 625-630, 1910.

[22] Wang C.H., Brown, M.W. Life Prediction Techniques for Variable Amplitude Multiaxial Fatigue - Part I: Theories Journal of Engineering Materials and Technology, 118, pp. 367-70, 1996.

[23] Langlais, T.E., Vogel J.H., Chase T.R. Multiaxial Cycle Counting for Critical Plane Methods International Journal of Fatigue, 25, pp. 641-47, 2003.

[24] Lee B.L., Kim K.S., Nam K.M. Fatigue Analysis Under Variable Amplitude Loading Using an Energy Parameter International Journal of Fatigue, 25, pp. 621-31, 2003.

[25] MSC.Nastran V70, Advanced Dynamics User's Guide. MSC.Software Coporation, Chapter 2, pp. 107-108, 2005.

[26] Papadopoulos, I.V., Davoli, P., Gorla, C., Filippini, M., Bernasconi, A. A comparative study of multi-axial high-cycle fatigue criteria for metals. Int. J. Fatigue, 19, pp. 219-35, 1997.

[27] Pitoiset, X., Preumont, A., Spectral methods for multi-axial random fatigue analysis of metallic structures. International Journal of Fatigue, 22, 541-50, 2000 .

[28] Fatemi, A., Yang, L., Cumulative fatigue damage and life prediction theories: a survey of the state of the art for homogeneous materials. Int. J. Fatigue, 20(1), pp. 9-34, 1998.

[29] Corten, H. T. and Dolon, T. J., Cumulative fatigue damage. In: Proceedings of the International Conference on Fatigue of Metals, Institution of Mechanical Engineering and American Society of Mechanical Engineers, pp. 235-46, 1956.

[30] Freudenthal, A. M. and Heller, R. A., On stress interaction in fatigue and a cumulative damage rule. Journal of the Aerospace Sciences, 26(7), pp. 431-42, 1959 .

[31] Sarkani S., Michaelov G., Kihl D.P., Beach J.E., Fatigue of Welded Steel Joints under Wideband Loadings Probabilistic Engineering Mechanics, 11(4), pp. 2217, 1996.

[32] Agerskov, H., Fatigue in Steel Structures Under Random Loading Journal of Constructional Steel Research, 53, pp. 283-305, 2000. 


\section{Appendix}

\section{A Finite element analysis of random vibrations}

It is well know that the cross-power spectral density $S_{x_{i} x_{j}}$ of any couple of degrees of freedom can be expressed as

$$
S_{x_{i} x_{j}}(\nu)=\sum_{r=1}^{n} \sum_{s=1}^{n} u_{i r} u_{j s} \tilde{H}_{r}(\nu) S_{f_{r} f_{s}}(\nu) H_{s}(\nu)
$$

where $u_{i r}$ is the $i$-th component of the $r$-th discretized vibration mode, $\tilde{H}_{r}(\nu)$ is the complex conjugate of the harmonic response function associated to the $r$-th mode and

$$
S_{f_{r} f_{s}}(\nu)=\frac{1}{\omega_{r}^{2}} \frac{1}{\omega_{s}^{2}} \sum_{p=1}^{n} \sum_{q=1}^{n} u_{r p} u_{s q} S_{F_{p} F_{q}}(\nu)
$$

is the cross-PSD of the $r$-th and $s$-th modal force/moment, where $\omega_{r}=2 \pi \nu_{r}$ is the $r$-th natural angular frequency and $S_{F_{p} F_{q}}$ is the cross-PSD of the $p$-th and $q$-th external excitations.

Let $\epsilon(t)$ be the vector containing the components of the strain tensor so that

$$
\epsilon^{T}=\left[\begin{array}{llllll}
\epsilon_{x x} & \epsilon_{y y} & \epsilon_{z z} & \gamma_{x y} & \gamma_{x z} & \gamma_{y z}
\end{array}\right]
$$

and similarly let $\sigma(t)$ be the vector comprising the components of the stress tensor

$$
\sigma^{T}=\left[\begin{array}{llllll}
\sigma_{x} & \sigma_{y} & \sigma_{z} & \tau_{x y} & \tau_{x z} & \tau_{y z}
\end{array}\right]
$$

In the FE method the strain field within an element is obtained by writing

$$
\epsilon^{(e)}=\mathbf{B}^{(e)}(x, y, z) \delta^{(e)}
$$

where $(x, y, z)$ are the spatial coordinates, $\mathbf{B}^{(e)}(x, y, z)$ is a $\left(6 \times m^{(e)}\right)$ matrix and $\delta^{(e)}$ is the $\left(m^{(e)} \times 1\right)$ vector of the elemental displacement/rotation having $m^{(e)}$ components. Both $\mathbf{B}^{(e)}(x, y, z)$ and $\delta^{(e)}$ depend on the kind of element considered and the shape functions employed. Of course $m^{(e)}$ as well as $\mathbf{B}^{(e)}(x, y, z)$ may vary from element to element depending on the local mesh topology. Similarly for the stress field the following equation holds

$$
\sigma^{(e)}=\hat{\mathbf{C}}^{(e)} \mathbf{B}^{(e)}(x, y, z) \delta^{(e)}
$$


where $\hat{\mathbf{C}}^{(e)}$ is the $(6 \times 6)$ constitutive tensor for the material the element is made of. Let $\mathbf{A}^{(e)}$ be a matrix which extracts from the global vector of displacements only those featuring the element $(e) ; \mathbf{A}^{(e)}$ is a $\left(m^{(e)} \times n\right)$ matrix, since the element are supposed to have $m^{(e)}$ nodal parameters and the whole model has $n$ degrees of freedom. The extraction matrix has either zero or unit entries. The $e$-th element displacement/rotations are obtained as

$$
\delta^{(e)}=\mathbf{A}^{(e)} x
$$

Therefore the elemental strains and stresses can be expressed as

$$
\begin{aligned}
\epsilon^{(e)} & =\mathbf{B}^{(e)}(x, y, z) \mathbf{A}^{(e)} x \\
\sigma^{(e)} & =\hat{\mathbf{C}}^{(e)} \mathbf{B}^{(e)}(x, y, z) \mathbf{A}^{(e)} x
\end{aligned}
$$

Employing the last equation it is easy to show that the cross-PSD of the stress components within an element $e$ is given by

$$
S_{\sigma_{i} \sigma_{m}}^{(e)}(\nu)=\sum_{l=1}^{n} \sum_{q=1}^{n} D_{i l m q}^{(e)} S_{x_{l} x_{q}}(\nu)
$$

where

$$
D_{i l m q}^{(e)}=\sum_{j=1}^{n} \sum_{k=1}^{n} \sum_{n=1}^{n} \sum_{p=1}^{n} \hat{C}_{i j}^{(e)} \hat{C}_{m n}^{(e)} B_{j k}^{(e)} B_{n p}^{(e)} A_{k l}^{(e)} A_{p q}^{(e)}
$$

is a matrix which depends on the spatial coordinates $(x, y, z)$. Therefore the cross-PSD of the elemental stress (A.10) can be obtained from that of the degrees of freedom (A.1) representing the discretized structure.

The theory of random vibration here summarized allows describing the structural response to distributed external forces; however the specific problem under investigation requires estimating the response of the structure to uniform translational/rotational accelerations. This can be done by assuming a rigid connection of the structure to a seismic mass, i.e. a relatively large mass element to which the accelerations are applied. Thus the motion of the structure follows that of the seismic mass and the displacements, strains and stresses are expressed in a rigid reference frame attached to the seismic mass and to the structure. Therefore the force inputs to the structure are only those featuring the acceleration of the seismic mass; all the other nodal external forces/moments have to be set to zero. The overall deformation of the structure depends only on its inertia properties. Therefore the external excitation $F(t)$ applied to the structure can be expressed as follows

$$
F(t)=M_{*} \mathbf{G} a
$$


where $M_{*}$ is a scalar quantity representing the seismic mass, $\mathbf{G}$ is a $(n \times 6)$ matrix and $a$ is the $(6 \times 1)$ acceleration vector applied to the seismic mass, i.e.

$$
a^{T}=\left[\begin{array}{llllll}
g_{x} & g_{y} & g_{z} & \phi_{x} & \phi_{y} & \phi_{z}
\end{array}\right]
$$

where the translational acceleration components are given by the "g" components and the rotational ones by the " $\phi$ " terms. The matrix $\mathbf{G}$ has either zero or unity entries, in order to apply the specific acceleration components to the degrees of freedom representing the seismic mass motion.

As a consequence of the previous assumptions, the cross-PSD of the external excitations can be expressed as

$$
S_{F_{i} F_{k}}(\nu)=M_{*}^{2} G_{i j} G_{k l} S_{a_{j} a_{l}}(\nu)
$$

where $S_{a_{j} a_{l}}$ is the cross-PSD of the external accelerations. Note that in (A.14) there is no summation on $j$ and $l$, since each degree of freedom associated to the seismic mass is related only to one component of the external acceleration. Therefore substituting (A.14) via (A.2) into the displacement cross-PSD (A.1) and this latter into the elemental stress cross-PSD (A.10) yields the explicit relation which holds between acceleration and elemental stresses

$$
S_{\sigma_{i} \sigma_{m}}^{(e)}(\nu)=\sum_{l=1}^{n} \sum_{q=1}^{n} D_{i l m q}^{(e)}(x, y, z) \sum_{r=1}^{n} \sum_{s=1}^{n} \sum_{j=1}^{n} \sum_{k=1}^{n} U_{l q r s j k}(\nu) G_{j t} G_{k u} S_{a_{t} a_{u}}(\nu)(\mathrm{A}
$$

where

$$
U_{l q r s j k}(\nu)=M_{*}^{2} \frac{1}{\omega_{r}^{2}} \frac{1}{\omega_{s}^{2}} u_{l r} u_{q s} u_{r j} u_{s k} \tilde{H}_{r}(\nu) H_{s}(\nu)
$$

Eq. (A.15) can be written in matrix form as follows

$$
\mathbf{S}_{\sigma \sigma}^{(e)}(\nu)=\mathbf{D}^{(e)}(x, y, z) \mathbf{U}(\nu) \mathbf{G S}_{a a}(\nu) \mathbf{G}^{T}
$$

The stress cross-PSD in eq. (A.17) seems to be related to the magnitude of the seismic mass $M_{*}$. This is not true, since the free response eigenvectors are normalized versus the mass matrix $\mathbf{M}$ : if the seismic mass $M_{*}$ is some order of magnitudes larger than any other mass in the structure, it also represents the dominant term in the overall mass matrix $\mathbf{M}^{1}$. Thus, due to the normalization, the eigenvector components are inversely proportional to $\sqrt{M_{*}}$ and so (A.17) is independent from the $M_{*}$.

\footnotetext{
$\overline{1}$ The rotational inertia terms are proportional to the seismic mass module $M_{*}$ multiplied by a properly scaled squared gyration radius.
} 